\title{
p5 Peptide-Loaded Human Adipose-Derived Mesenchymal Stem Cells Promote Neurological Recovery After Focal Cerebral Ischemia in a Rat Model
}

\author{
Arjun Paudyal $^{1,2}$. Flavia Semida Ghinea ${ }^{3}$ - Mircea Popescu Driga ${ }^{3} \cdot$ Wen-Hui Fang ${ }^{1} \cdot$ Giulio Alessandri $^{4}$. \\ Laura Combes $^{1} \cdot$ Hans Degens ${ }^{1,5,6} \cdot$ Mark Slevin $^{1,5,7} \cdot$ Dirk M. Hermann $^{8} \cdot$ Aurel Popa-Wagner ${ }^{4,8,9}$
}

Received: 30 January 2020 / Revised: 6 March 2020 / Accepted: 10 March 2020 / Published online: 6 May 2020

(C) The Author(s) 2020

\begin{abstract}
Adipose-derived mesenchymal stem cells markedly attenuated brain infarct size and improved neurological function in rats. The mechanisms for neuronal cell death have previously been defined in stress states to suggest that an influx of calcium ions into the neurons activates calpain cleavage of $\mathrm{p} 35$ into $\mathrm{p} 25$ forming a hyperactive complex that induces cell death. Now we report that p5, a 24-residue peptide derived from $\mathrm{p} 35$, offers protection to neurons and endothelial cells in vitro. In vivo administration of human adipose-derived mesenchymal stem cells (hADMSCs) loaded with this therapeutic peptide to post-stroke rats had no effect on the infarct volume. Nevertheless, the treatment led to improvement in functional recovery in spatial learning and memory (water maze), bilateral coordination and sensorimotor function (rotating pole), and asymmetry of forelimb usage (cylinder test). However, the treatment may not impact on cutaneous sensitivity (adhesive tape removal test). In addition, the double immunofluorescence with human cell-specific antibodies revealed that the number of surviving transplanted cells was higher in the periinfarcted area of animals treated with hADMSCs + P5 than that in hADMSC-treated or control animals, concomitant with reduced number of phagocytic, annexin3-positive cells in the peri-infarcted region. However, the combination therapy did not increase the vascular density in the peri-infarcted area after stroke. In conclusion, administration of hADMSC-loaded p5 peptide to post-stroke rats created conditions that supported survival of drug-loaded hADMSCs after cerebral ischemia, suggesting its therapeutic potential in patients with stroke.
\end{abstract}

Keywords Cyclin-dependent kinase $5 \cdot$ Peptide $5 \cdot$ Neuroprotection $\cdot$ Human adipose-derived mesenchymal stem cells $\cdot$ Cerebral ischemia $\cdot$ Rat

Arjun Paudyal, Flavia Semida Ghinea, Mircea Popescu Driga and WenHui Fang contributed equally to this work.

Electronic supplementary material The online version of this article (https://doi.org/10.1007/s12975-020-00805-0) contains supplementary material, which is available to authorized users.

Mark Slevin

M.A.Slevin@mmu.ac.uk

$\triangle$ Aurel Popa-Wagner

aurel.popa-wagner@geriatrics-healthyageing.com

1 Department of Life Sciences, Faculty of Science and Engineering, Manchester Metropolitan University, Chester Street, Manchester, UK

2 Department of Human Movement Sciences, Faculty of Behavioural and Movement Sciences, Vrije University Amsterdam, Amsterdam Movement Sciences, Amsterdam, The Netherlands

3 Doctoral School, Department of Center of Clinical and Experimental Medicine, University of Medicine and Pharmacy Craiova, Craiova, Romania
4 Cellular Neurobiology Laboratory, Department of Cerebrovascular Diseases, IRCCS Neurological Institute C. Besta, 20133 Milan, Italy

5 University of Medicine and Pharmacy, Targu Mures, Romania

6 Lithuanian Sports University, Kaunas, Lithuania

7 Institute of Dementia and Neurological Aging, Weifang Medical University, Weifang, China

8 Department of Neurology Chair of Vascular Neurology and Dementia, University of Medicine Essen, Essen, Germany

9 Griffith University Menzies Health Institute of Queensland, Gold Coast Campus, Gold Coast Campus QLD 4222, Australia 


\section{Introduction}

Therapeutic standard procedure for ischemic stroke is cerebral artery recanalization with tissue-type plasminogen activator (tPA). However, brain inflammation and comorbidities have a negative impact on successful revascularization and functional outcome [1-5].

Numerous studies have shown that bone marrow mesenchymal stem cell (BMSC) transplantation could foster the recovery of neurological function and reduce infarct size in rats with acute ischemic stroke [6, 7]. In the animal model of cerebral ischemia, BMSC transplantation could improve neurological functional outcomes and decrease the infarction size via differentiation, replacement, and neural circuit reconstruction by enhancing angiogenesis [8]. Further, adipose-derived mesenchymal stem cells markedly attenuated brain infarct size and improved neurological function in rats [9].

Combinations of MSCs and the peptide granulocyte colony-stimulating factor (G-CSF), given separately, to poststroke aged rats led to a robust and consistent improvement of neurological function after 28 days [10]. The combination therapy also led to robust angiogenesis in the formerly infarct core and in islets of the soft tissue beyond [11].

Cyclin-dependent kinase 5 (CDK5), a serine/threonine kinase, in complex with its activators, p35 (protein of $35 \mathrm{kDa}$ ) and p39 (protein of $39 \mathrm{kDa}$ ), is essential for early neurodevelopment in mammals [12]. However, a variety of neurotoxic conditions, such as ischemic brain damage, oxidative stress, amyloid- $\beta$ peptide $(A \beta)$, excitotoxicity, calcium dyshomeostasis, and inflammation, induce influx of $\mathrm{Ca}^{2+}$ ions and the rise in the intracellular $\mathrm{Ca}^{2+}$, thereby promoting activation of calpain, a $\mathrm{Ca}^{2+}$-activated protease, which in turn cleaves p35 into p25 and a p10 fragment $[13,14]$. p25 forms a more stable CDK5-p25 hyperactive complex, which causes aberrant hyperphosphorylation of various substrates of CDK5 like tau and neurofilament, that leads to neuronal apoptosis and is associated with neuropathology. Therefore, a therapeutic approach directed specifically at CDK5-p25 complex might prove successful. To improve its therapeutic efficacy, several peptides consisting of amino acid residues of $\mathrm{p} 35$, such as CDK5 inhibitory peptide (CIP, a peptide of 125 amino acid residues), p10, and $\mathrm{p} 5$, have been generated and proven to specifically reduce CDK5-p25 increased activity without affecting the normal endogenous CDK5-p35 or other CDK activities [15-21]. In particular, p5 also reduced neuronal apoptosis induced by $\mathrm{Ca}^{2+}$ in hypoxia/ ischemia brain injury [22, 23].

Recently, BMSCs have been used as vectors in cancer therapy by delivering oncolytic viruses and drug-loaded nanoparticles [15]. Moreover, a recent study has shown that MSC given through their natural container (MFAT) led to an improved MSC survival and therefore may prolong the delivery of the molecule/proteins transported [16]. However, the use of
BMSCs as drug carrier of the CIP peptide for stroke has not been tested. In this work, we hypothesized that a prolonged delivery of the CIP peptide by fat-derived MSC may lead to improved post-stroke recovery by blocking neuronal and endothelial cell apoptosis through CDK5-p35 and subsequent p53 activation [17]. This pathway was previously shown to be a key initiator of cellular apoptosis following stroke, associated with excitotoxic release of calpains and subsequent hyper-phosphorylation of the CDK5 protein following p35 conversion to $\mathrm{p} 25[18,19]$.

\section{Methods}

\section{Cell Culture}

Human adipose-derived mesenchymal stem cells (hADMSCs) were provided by Professor Giulio Alessandri and Professor Valentina Ceserani. hADMSCs were isolated from peri-umbilical fat tissue and characterized as described [20]. Human neuroblastoma neuroblastic type SH-SY5Y cells and bovine aortic endothelial cells (BAECs) were cultured in Dulbecco's modified Eagle's medium (DMEM; Lonza) supplemented with $10 \%$ FBS and $1 \%$ L-glutamine.

\section{p5 Priming of hADMSCs}

$\mathrm{p} 5$, a 24-residue peptide, derived from $\mathrm{p} 35$, the CDK5 activator, was chemically synthesized with 5-FAM conjugated on the N-terminus and KRKR-KRKR wrapper on the C-terminus [15] (Cambridge Research Biochemicals). The sequence of $\mathrm{p} 5$ p e p t i d e i s [5-F A M ] - K E A F - W DRCLSVINLMSSKMLQINAKRKRKRKR-amide. The toxicity of p5 on hADMSCs was determined in a 24-h alamarBlue assay (cytotoxicity test; Life Technologies) and in a 3-day alamarBlue assay (anti-proliferative test). Based on these results, the p5 priming of hADMSCs was carried out with two concentrations, $25 \mu \mathrm{g} / \mathrm{ml}$ and $100 \mu \mathrm{g} / \mathrm{ml}$.

\section{In Vitro Proliferation Assay}

The protective effect of both $\mathrm{p} 5$ or $\mathrm{CM}$ of $\mathrm{p} 5$ primed hADMSCs on SH-SY5Y cells and BAEC proliferation after the treatment of $\mathrm{Ca}^{2+}$ ionophore combined with $\mathrm{CaCl}_{2}$ was determined by the alamarBlue assay (Life Technologies). Briefly, $7.0 \times 10^{4}$ SH-SY5Y cells or $1.0 \times 10^{3}$ BAECs were plated in 96-well multiwell plates in DMEM. The cells were then treated with $5 \mu \mathrm{M}$ calcium ionophore A23187 (SigmaAldrich) and $2.5 \mathrm{mM} \mathrm{CaCl}_{2}$ (Sigma-Aldrich) with or without p5 or CM of p5-primed hADMSCs. Three wells were devoted for the assay every day for 3 days, and time course of cell proliferation was monitored. 


\section{Animals and Experimental Design}

The subjects of these experiments were male Sprague-Dawley rats ( $n=40 ; 3-4$ months of age) 320-400 g kept under standard laboratory conditions with free access to food and water. The numbers reported in the results refer to the number of animals that survived the surgery and completed the 4-week testing period. All appropriate measures were taken to minimize pain and suffering.

\section{Randomization}

A scientist was in charge of randomization by (i) group assignment; (ii) behavioral testing; (iii) surgery assignment; and (iv) treatment assignment.

\section{Behavioral Testing}

To evaluate changes in neurological function associated with ischemia, the rats were subjected to a variety of somatosensory, motor, learning, and memory tests before and after surgery. All testing was performed from 09:00 to 11:00. Results obtained before surgery were used to define $100 \%$ functionality for each animal on each test, and functional recovery was expressed as percent recovery relative to the pre-surgery baseline.

\section{Bilateral Sensorimotor Coordination: Rotating Beam-Walking Test}

The rotating pole task assesses coordination and sensorimotor function in the middle cerebral artery occlusion (MCAO) model. Each rat was tested for its ability to cross a rotating (6 rpm) horizontal rod. The score assessment was done as previously described $[10,21]$. Briefly, the time taken for the rat to traverse the rotating cylinder and join a group of rats visible at the finish line was measured. The score assessment was twofold: (i) time (s) required to traverse the rotating cylinder and (ii) the score as follows: $0=$ rat falls immediately (onto a soft surface); 1 = rat does not walk forward, but stays on the rotarod; 2 = rat walks, but falls before reaching the goal; $3=$ rat traverses the rod successfully, but the limbs are used asymmetrically; $4=$ the left hindlimb is used less than $50 \%$ of the time taken to traverse the rod; $5=$ the rat successfully traverses the rod, but with some difficulties; $6=$ no mistakes, symmetric movements.

\section{Asymmetric Sensorimotor Deficit: Adhesive Tape Removal Test (Tape Test) and Cylinder Test}

We assessed the asymmetry of sensorimotor deficit of the forelimbs induced by unilateral MCAO, by tape test and cylinder test as previously described [10, 21]. For the cylinder test, the forelimb use asymmetry during vertical exploration of the walls of a $20-\mathrm{cm}$-diameter and $40-\mathrm{cm}$-height glass cylinder was measured. Rats which did not make at least 20 exploratory contacts with the walls were excluded from the analysis procedure. The asymmetry index was calculated as (Right Left)/(Right + Left), where Right and Left are the number of contacts of the right.

\section{Spatial Learning and Memory: Morris Water Maze}

The Morris water maze task was used to assess spatial learning and memory. One week before surgery, aged rats were trained to find a submerged platform in a large (180-cm-diameter) pool filled to within $20 \mathrm{~cm}$ of the upper edge with water maintained at $26{ }^{\circ} \mathrm{C}$ as previously described [10]. The swim path was recorded by an image analysis system (Noldus, Holland) that computed path length and percentage of time spent in each of the four quadrants.

\section{Surgery}

Cerebral infarction was induced by transcranial interruption of blood flow by transiently lifting the middle cerebral artery (MCA) with a tungsten hook as previously described [19]. Throughout surgery, anesthesia was maintained by spontaneous inhalation of $2 \%$ isoflurane in a mixture of $70 \%$ nitrous oxide and $30 \%$ oxygen. After $90 \mathrm{~min}$, the tungsten hook was released and the common carotid arteries were re-opened. Surgery was performed under antiseptic conditions to minimize the risk of infection. The rats then received $0.05 \mathrm{mg} / \mathrm{kg}$ SC buprenorphine (sc) for treatment of post-operative pain.

Subsequent to survival times of 28 days, the rats were deeply anesthetized and perfused with neutral buffered saline followed by buffered $4 \%$ freshly depolymerized paraformaldehyde. The brain was removed, post-fixed in $4 \%$ buffered paraformaldehyde for $24 \mathrm{~h}$, cryoprotected in $15 \%$ glycerol prepared in $10 \mathrm{mM}$ phosphate-buffered saline, flash-frozen in isopentane, and stored at $-70{ }^{\circ} \mathrm{C}$ until sectioning.

\section{Treatments}

The contents of the frozen vials containing hADMSCs are resuspended in a T75 flask containing $10 \mathrm{ml}$ of stem cell medium (SCM). After a period of about $24 \mathrm{~h}$, the media was removed and replaced with fresh SCM primed with the therapeutic peptide $\mathrm{p} 5$ at a final concentration of $10 \mu \mathrm{g} / \mathrm{ml}$. After an incubation period of $24 \mathrm{~h}$, the cells were washed 3 times with PBS before trypsinization. One group of rats $(n=8)$ was treated with $1 \times 10^{6} \mathrm{hADMSCs}$, given intracortically at 3 different locations in the exposed area proximal to the occluded middle cerebral artery, immediately after stroke. The second group $(n=8)$ was treated with hADMSCs $+\mathrm{p} 5$ and the control $(n=8)$ group was treated with the vehicle. 


\section{Determination of Infarct Volume by Immunohistostaining}

To assess the size of the infarct induced by focal ischemia, we used mouse anti-NeuN immunostaining [10]. Every 20th freefloating section of $25 \mu \mathrm{m}$ was immunostained for NeuN to cover the entire infarcted volume, which was then calculated as the sum of the partial areas using ImageJ.

\section{Collagen IV Immunofluorescence}

Sections (25- $\mu \mathrm{m}$-thick) were cut on a freezing microtome and processed for immunohistochemistry as previously described [10]. Briefly, tissue sections were exposed overnight at $4{ }^{\circ} \mathrm{C}$ to rabbit anti-collagen IV (1:2000, Abcam, UK) and the signal was amplified utilizing an anti-rabbit polymer-based secondary detection system (Histofine polymer-HRP, Nichirei, Japan) diluted 1:10 in PBS containing 1\% normal goat serum and $0.3 \%$ Tween 20 .

\section{HuNu/CD105 Double Immunofluorescence}

For phenotyping, the tissue was incubated with a mix of rabbit anti-mouse $\mathrm{HuNu}(1: 1000$, Novus Biologicals, UK) and mouse anti-human CD105 (1:1000, antibodies-on-line, Aachen, Germany) at $4{ }^{\circ} \mathrm{C}$ overnight. Next day, sections were incubated with a mix of Alexa Fluor ${ }^{\circledR} 488$ goat anti-rabbit IgG and Alexa Fluor® 555 goat anti-mouse IgG for CD105.

\section{Annexin A3/NeuN/lbal Triple Immunofluorescence}

Cryostat, free-floating sections of $25 \mu \mathrm{m}$ were fixed in $4 \%$ paraformaldehyde for $15 \mathrm{~min}$ and then washed extensively with PBS and incubated serially with the following primary antibodies, rabbit anti-AnxA3 (1:500, Abcam, UK), goat antiIbaI (1:3000, Abcam, UK), and mouse anti-NeuN (1:1000, Millipore, Germany). Secondary reagents were donkey antimouse Alexa 488, donkey anti rabbit-Alexa 568, and donkey anti goat-Alexa 647.

\section{Cell Counting of Co-localized Cells}

The number of co-labeled cells ( $\mathrm{HuNu}+\mathrm{CD} 105$-positive) and (AnxA3/IbaI) cells at the reperfusion times of 28 days was determined by counting cells on every tenth section in systematic random series across the entire infarcted volume as previously described [10].

\section{Quantitation of Microvascular Density}

Microvascular density was quantitated using the "hot spot" analysis. Briefly, hot spots, i.e., regions with a high density of collagen IV-positive microvessels, were identified using a $\times 40$ objective and were then counted using $\times 20$ objective, corresponding to a microscopic field of $0.7386 \mathrm{~mm}^{2}$ as previously described [11].

\section{Statistical Analyses}

Statistical analysis was performed using SPSS (V 22.0). Behavioral data were analyzed using two-way ANOVA (treatment $\times$ duration) with post-stroke time as a repeated measurement factor. The Greenhouse-Geisser correction was applied where the sphericity was violated. $T$ tests were performed for the single parameter comparison, and unequal variance between groups was assumed. All values are presented as mean \pm SEM. $p$ values $<0.05$ were considered statistically significant.

\section{Results}

\section{p5-Primed HADMSCs Protected Neurons and Endothelial Cells from Calpain-Induced Toxicity}

As the $\mathrm{Ca}^{2+}$ ionophore and $\mathrm{CaCl}_{2}$ treatment activated apoptosisrelated genes, such as p53 and caspase-3, we have examined the cytotoxic effects of the combined treatment of $\mathrm{Ca}^{2+}$ ionophore and $\mathrm{CaCl}_{2}$ on neurons (SH-SY5Y cells) and endothelial cells (BAECs). Compared to the untreated control cells, the 10-min treatment with $\mathrm{Ca}^{2+}$ ionophore and $\mathrm{CaCl}_{2}$ caused the immediate cell death on SH-SY5Y cells on day 1 (Fig. 1). Furthermore, the $\mathrm{Ca}^{2+}$ ionophore and $\mathrm{CaCl}_{2}$ treatment inhibited BAEC growth through 3-day culture, resulting in $71.4 \%$ reduction on day 3 although the $\mathrm{Ca}^{2+}$ ionophore and $\mathrm{CaCl}_{2}$ treatment did not affect the SH-SY5Y cell proliferation (Fig. 1).

We then investigated whether $\mathrm{p} 5$ or p5-primed hADMSCs would protect SH-SY5Y and endothelial cells (BAECs) against the cytotoxicity induced by $\mathrm{Ca}^{2+}$ ionophore and $\mathrm{CaCl}_{2}$ treatment.

The immediate cytotoxic assay on day 1 and proliferation assay on the following 2 days showed that neither $\mathrm{p} 5$ nor $\mathrm{CM}$ of untreated hADMSCs affected the SH-SY5Y cells or BAEC numbers with the 10-min treatment of $\mathrm{Ca}^{2+}$ ionophore and $\mathrm{CaCl}_{2}$, although $\mathrm{p} 5$ alone provided the slight protection in SH-SY5Y cells against immediate cytotoxicity induced by $\mathrm{Ca}^{2+}$ ionophore and $\mathrm{CaCl}_{2}$ treatment (Fig. 1). However, compared with the treatment of $\mathrm{Ca}^{2+}$ ionophore and $\mathrm{CaCl}_{2}$ alone, SH-SY5Y cell numbers in CM from $100 \mu \mathrm{g} / \mathrm{ml} \mathrm{p5-primed}$ hADMSCs collected on day 1 and day 2 and CM from $25 \mu \mathrm{g} / \mathrm{ml} \mathrm{p} 5$-primed hADMSCs collected on day 1 significantly increased by $22.8 \%, 25.3 \%$, and $25.6 \%$, respectively, with immediate cytotoxic assay on day 1 . Therefore, the CM of $\mathrm{p} 5$-primed hADMSCs provided better protection on neurons than endothelial cells against the immediate cytotoxicity induced by the calpain activation. 
SH-SY5Y Cells

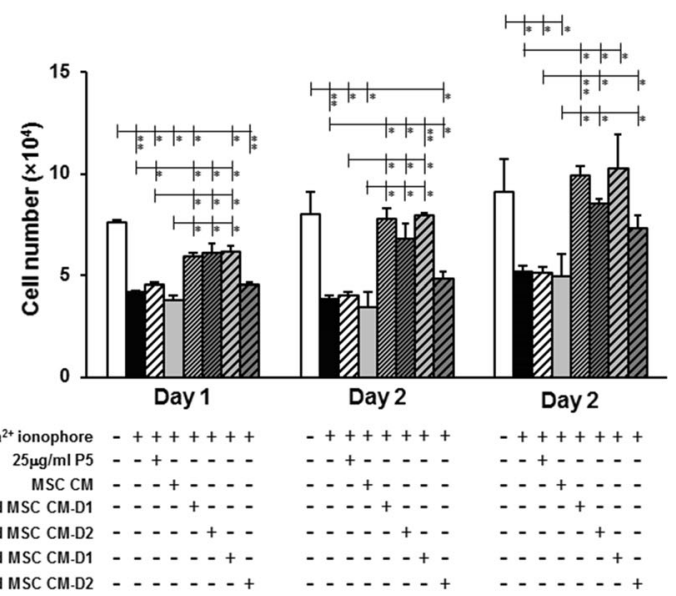

BAECS

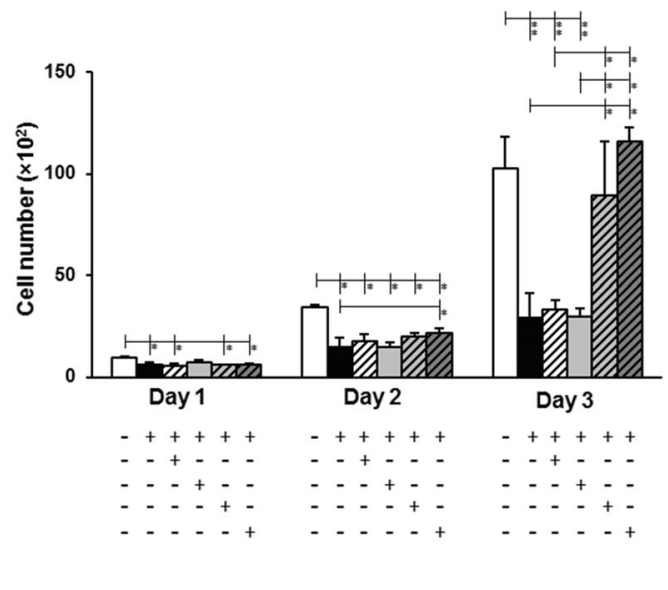

Fig. 1 The conditioned medium (CM) from p5-primed hADMSCs protected human neurons (SH-SY5Y cells) and endothelial cells (BAECs) from calpain-induced cytotoxicity. The cultured SH-SY5Y cells and BAECs were treated with $\mathrm{p} 5$ or $\mathrm{CM}$ from $100 \mu \mathrm{g} / \mathrm{ml}$ or $25 \mu \mathrm{g} / \mathrm{ml} \mathrm{p} 5$-primed hADMSCs collected on day 1 and day 2 for $1 \mathrm{~h}$, then further treated with $5 \mu \mathrm{M}$ calcium ionophore A23187 and $2.5 \mathrm{mM}$

More encouragingly, the $\mathrm{CM}$ from $100 \mu \mathrm{g} / \mathrm{ml} \mathrm{p} 5$-primed hADMSCs completely restored the cell growth in both neurons and endothelial cells after the treatment of $\mathrm{Ca}^{2+}$ ionophore and $\mathrm{CaCl}_{2}$ with the similar cell numbers as those in the untreated control group on day 3 .

\section{Behavioral Testing}

The experimental design and time flow of treatment and testing are shown in Fig. 2. To facilitate feeding during the first 3 days post-stroke, all animals were fed with moistened, soft pellets. The mortality rates were almost identical, $14 \%$ for each group. All deaths occurred between day 3 and day 10 post-stroke.

\section{Bilateral Sensorimotor Coordination: Rotating Pole Test}

At baseline, rats traversed the rod within $3 \mathrm{~s}$ with no mistakes (score of 6) and no differences in performance were observed between rats assigned to control and treatment groups (Fig. 2a). On day 4 following MCAO, for both rotating speeds, the performance on the rotating beam was impaired in all groups but both treatment groups showed significant improvement over the control group $(p<0.05)$ (Fig. 2a, b). Thereafter, the rats treated with the combination $\mathrm{MSCs}+\mathrm{p} 5$ recovered increasingly better, particularly on day 28 (Fig. 2a) as compared to the control group. However, with increasing difficulty $(6 \mathrm{rpm})$, the significance of recovery of the bilateral sensorimotor coordination diminished (Fig. 2b). In addition, no significant interaction (treatment $\times$ duration) effect was found for both speeds in the rotating pole test.
$\mathrm{CaCl}_{2}$ for $10 \mathrm{~min}$. The cell proliferation was monitored for 3 consecutive days using the alamarBlue assay. Data are means \pm SD (error bars). Significance (denoted as an asterisk) was calculated compared to the control using one-way ANOVA with Bonferroni post-test analysis (with $* p<0.05 ; * *<<0.01, \quad=3$ )

\section{Asymmetric Sensorimotor Deficit: Adhesive Tape Removal Test}

The time course of changes in recovery pattern of control animals, animals treated with MSCs alone, and animals treated with $\mathrm{MSCs}+\mathrm{p} 5$ is shown in Fig. 2c. The score significantly dropped down from baseline to day 7 and significantly improved on day 21 and day $28(p<0.001)$; however, no significant interaction (treatment $\times$ duration) was found. Though the scores from the rats treated with the combination of MSCs and $\mathrm{p} 5$ were slightly improved over the other treatment or controls at all time points after stroke, they were not statistically significant.

\section{Asymmetric Sensorimotor Deficit: Cylinder Test}

The forelimb asymmetry index indicated a strong deviation to the right (ipsilateral to the injured hemisphere) in controls $(0.33 \pm 0.04)$ as compared to MSCs $+\mathrm{p} 5$-treated rats $(0.25 \pm$ 0.03 ) on day 7 post-stroke. In addition, both treatment groups showed significant improvement over the control group on day 28 post-stroke (control versus MSCs, control versus MSCs together with peptide; both $p<0.01$ ). No significant interaction effect was found between time and treatment factor (Fig. 2d).

\section{Water Maze Test}

The statistical analysis revealed that there was a significant reduction of treatments on time taken by rats to reach the submerged platform $(p=0.005)$. No significant effect of the interaction (time $\times$ treatment) was found, suggesting that the pattern of recovery related to spatial learning and memory was similar between different groups at different time points after stroke. 
Fig. 2 Experimental design (upper panel) and time course of behavioral recovery after treatment with mesenchymal cells and $\mathrm{p} 5$. a, b Functional recovery on a rotating pole at 3 and $6 \mathrm{rpm}$. $\mathbf{c}, \mathbf{d}$ Time course of changes in sensorimotor function recovery after stroke therapy according to adhesive tape removal and cylinder test. Asymmetry of forelimb usage for the postural weight support in rats after stroke therapy. e Time course of poststroke recovery of (spatial) learning and memory by using water maze test. Significance between groups is denoted as $* p<0.05 ; * * p<0.01$, compared to the control group. "a", significantly different from baseline

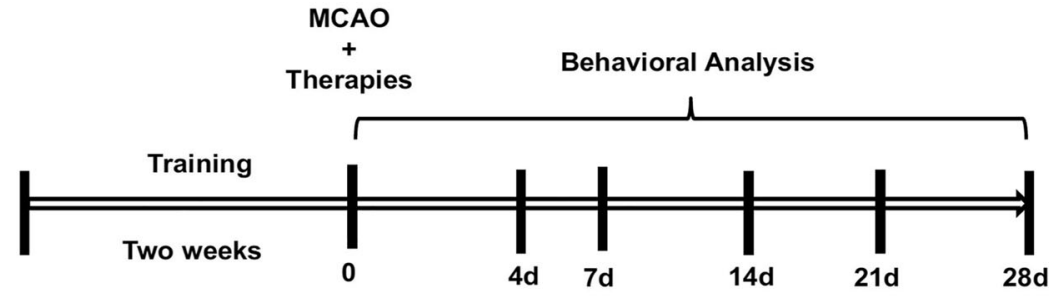

Tissue Analysis

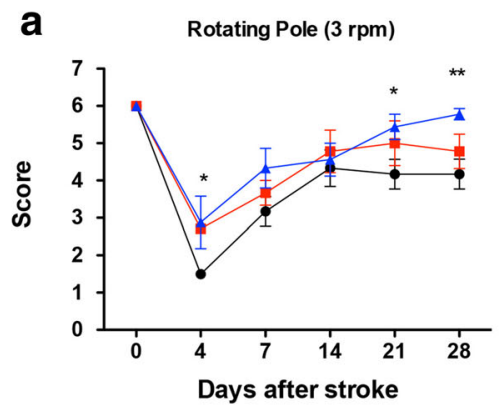

b

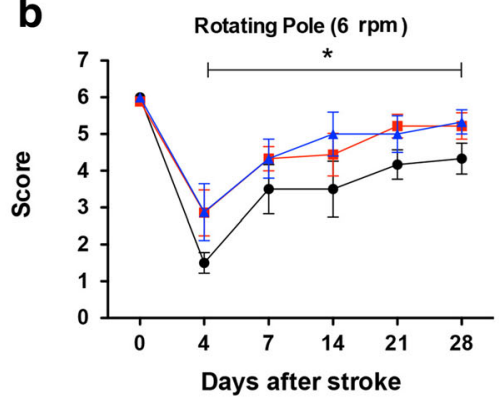

C

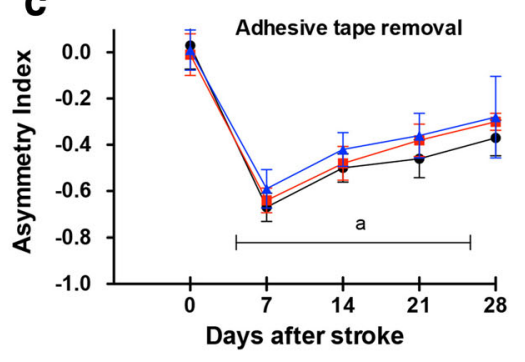

e

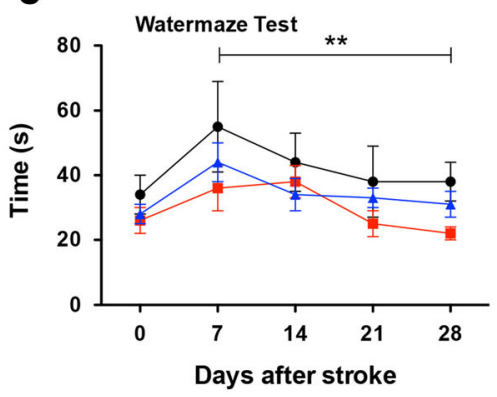

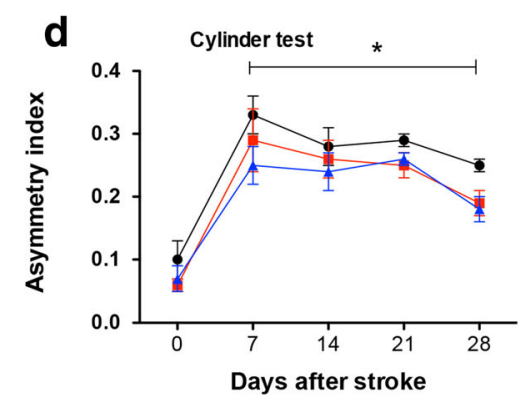

$\rightarrow$ Control

$\rightarrow$ MSC

$\leftarrow \mathrm{MSC}+\mathrm{p} 5$

\section{The Effect of Cell Therapy on the Infarct Volume}

Immunohistochemical staining of the infarct area on day 28 using an anti-NeuN antibody showed that the infarcted cortical volumes were largely similar and independent of treatment as compared to controls (Fig. 3). The anti-NeuN antibody sharply delineated the infarcted area.

\section{The Survival Rate of Transplanted Cells Was Higher in Animals Treated with hADMSCs + p5}

In the ipsilateral hemisphere, the injected human hADMSCs were detected in the perilesional area by double immunofluorescence with anti-human specific antibodies, $\mathrm{HuNu}$ (nuclear, green) and CD105 (cytoplasmic, red) (Fig. 4). We noted that in the peri-infarcted area of animals treated with hADMSCs + p5 (Fig. 4b, arrows), the number of surviving transplanted cells was 2.5-fold higher (Fig. 4c) than in hADMSC-treated animals (Fig. 4a).

\section{hADMSCs Loaded with p5 Reduced the Number of Inflammatory Cells in the Peri-infarcted Area}

Cell survival in the perilesional area after stroke is limited by an exaggerated inflammatory response. We asked if the neuroprotective effect of hADMSCs loaded with $\mathrm{p} 5$ might be a consequence of decreased number of inflammatory cells in the perilesional area. Indeed, by triple immunofluorescence phenotyping and cell counting, we found that the number of AnxA3/IbaI co-localized cells was decreased by $35 \%$ in 
Fig. 3 Stroke volumes by NeuN immunohistochemistry. By

immunohistochemistry at day 28 , the infarct volumes for controls (a), MSCs alone (b), and MSCs + p5 (c) were largely similar (e). NeuN immunohistochemistry sharply delineated the infarcted area (d). Cc corpus callosum, $\mathrm{Cx}$ cortex, IC infarct core

Fig. 4 The survival rate of transplanted cells was higher in animals treated with hADMSCs + p5. Note that in the peri-infarcted area of animals treated with hADMSCs + p5 (b, arrows), the number of surviving transplanted cells was 2.5-fold higher (d) than in hADMSC-treated (a) animals. The nuclei in the peri-infarcted area of vehicle-treated animals is shown in $\mathbf{c}\left({ }^{* * *} p=0.001\right)$
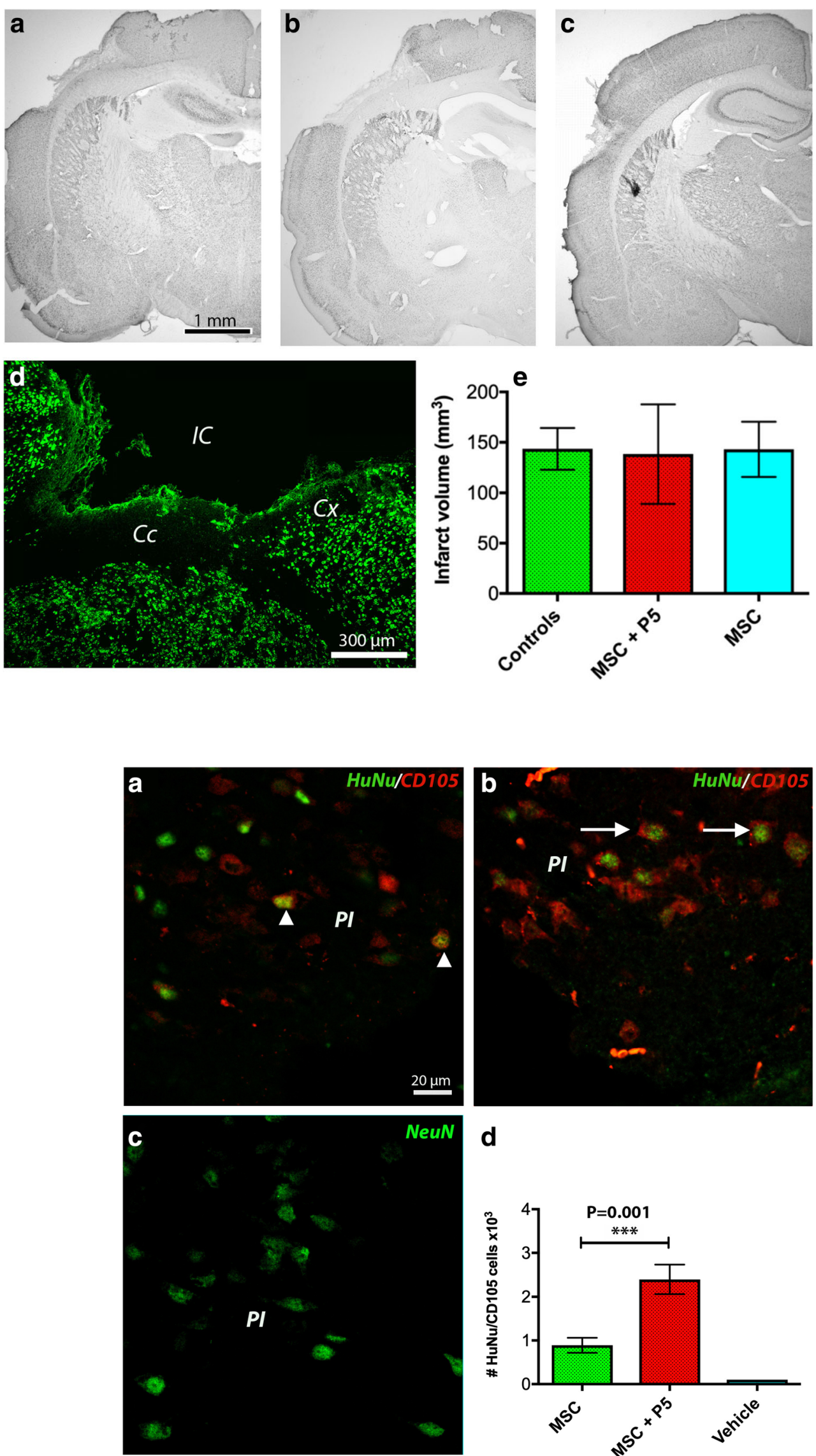

d

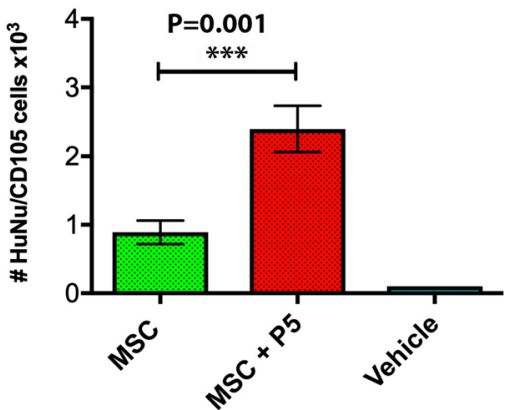


animals treated with hADMSCs + p5 (Fig. 5d). Occasionally, we found that microglial cells that were immunopositive for both IbaI and ANXA3 wrapped around neurons, highly suggesting an association of ANXA3 expression with a phagocytic phenotype (Fig. 5a-c).

\section{hADMSCs Loaded with p5 Did Not Increase Vascular Density in the Peri-infarcted Area}

Treatment with MSCs stimulates post-stroke angiogenesis [4, 8]. We asked if the neuroprotective effect of hADMSCs loaded with $\mathrm{p} 5$ might be a consequence of increased vascularity in the perilesional cortex. We found that the treatment with hADMSCs alone led to a significant increase (2.1-fold) in the vascular density in the peri-infarcted area as compared to control animals (Fig. 6a). Although the treatment of animals with hADMSCs + p5 (Fig. 6b) did also increase (2.3-fold) the vascular density in the peri-infarcted area, the increase was not significant (Fig. 6c, d).

\section{Discussion}

In ischemic stroke, the mechanisms for neuronal cell death has previously been defined in stress states to suggest that an influx of calcium ions into the neurons activates calpain cleavage of p35 into p25 forming a hyperactive complex that induces cell death. Now we report that $\mathrm{p} 5$ offers protection to both SH-SY5Y and BAEC cells. In vivo administration of hADMSCs loaded with the therapeutic peptide $\mathrm{p} 5$ to post- stroke rats led to improvement in functional recovery and increased number of surviving transplanted cells, most likely by reducing the number of inflammatory ANXA3/IbaIpositive cells in the peri-infarcted region.

Previous work has identified that $\mathrm{p} 5$ offers protection to both neurons and endothelial cells $[12,20]$. Indeed, this present study has demonstrated a specific protection towards neurons in vitro. We found that $\mathrm{p} 5$ offers protection to SH-SY5Y and BAEC cells, although the protection for SH-SY5Y cells was much more significant when compared to the control. Specifically, the data showed $\mathrm{p} 5$ protection for SH-SY5Y cells throughout the 3 days in all conditions, peaking on day 2, with continual significance to both control groups. This significant finding indicates the high affinity neurons have for $\mathrm{p} 5$. When comparing the protection of BAECs at no time point did $\mathrm{p} 5$ offer protection greater than the control group, and similarly to SY5Y cells, protection increased over time until day 2 when p5 25DRD1 and 25DRD2 displayed negative values of cell number. This infers that $\mathrm{p} 5$ at that concentration within the endothelial cell inhibited CDK5-p35 rather that CDK5-p25, suggesting that higher dosages are required for BAEC protection.

Surprisingly, the CM from $\mathrm{p} 5$-primed hADMSCs provided much stronger protection than $\mathrm{p} 5$ alone and $\mathrm{CM}$ of untreated hADMSCs did not show any protection against the calpain activation induced by the treatment of $\mathrm{Ca}^{2+}$ ionophore and $\mathrm{CaCl}_{2}$. These findings suggest that hADMSCs might release other trophic factors, which could work together with released p5 in the $\mathrm{CM}$, thereby providing more potent therapeutic effects.
Fig. 5 AnnexinA3/NeuN/IbaI triple immunofluorescence phenotyping and cell counting. In animals treated with hADMSCs + $\mathrm{p} 5$, the number of co-localized ANXA3/IbaI cells (arrowheads) was decreased by $35 \%$ (d). Occasionally, microglial cells that are immunopositive for both IbaI and ANXA3 wrapped around neurons (arrows), suggesting an association of ANXA3 expression with a phagocytic phenotype $(\mathbf{a}-\mathbf{c})(* * p=0.01)$
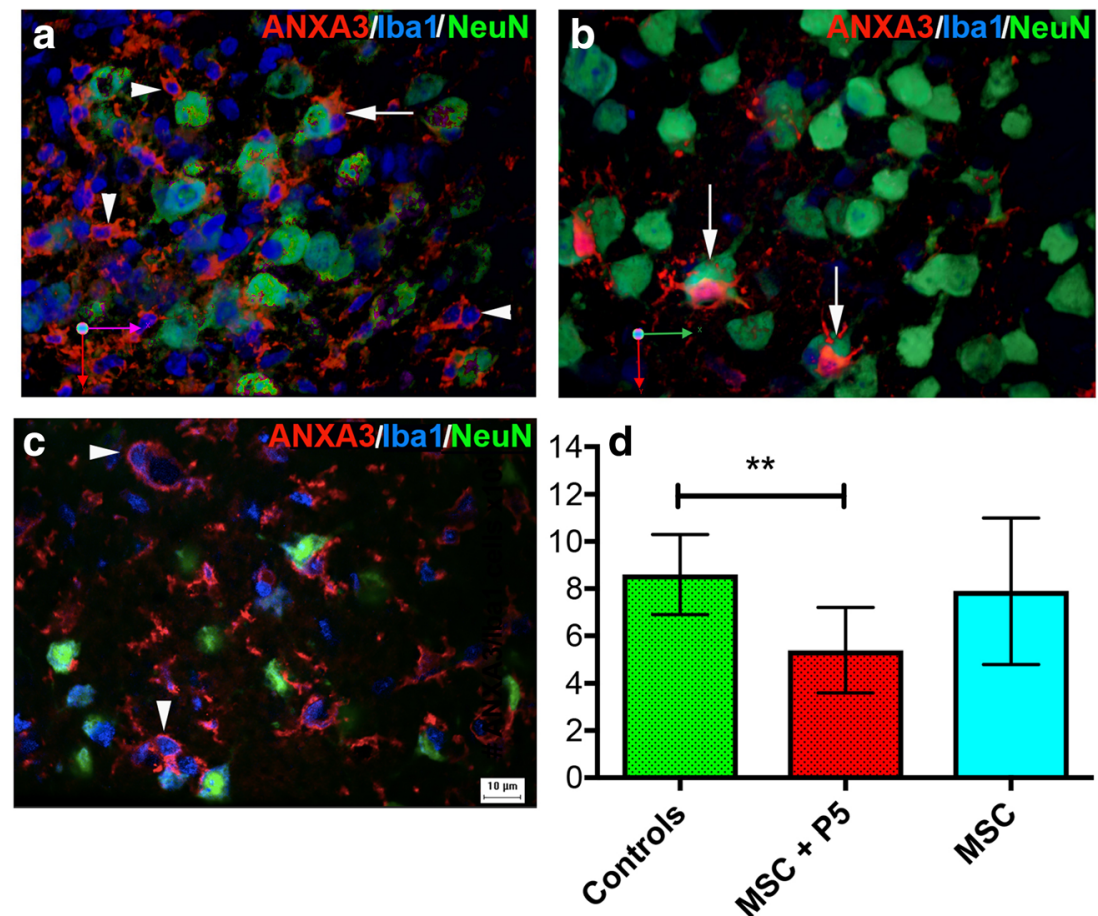
Fig. 6 Effect of treatment on post-stroke vascular density. Treatment with hADMSCs alone (b) or with hADMSCs + p5 (c) led to a significant increase (2.12.3 -fold) in the vascular density in the peri-infarcted area as compared to control animals (a) $(* * * p=0.001)$
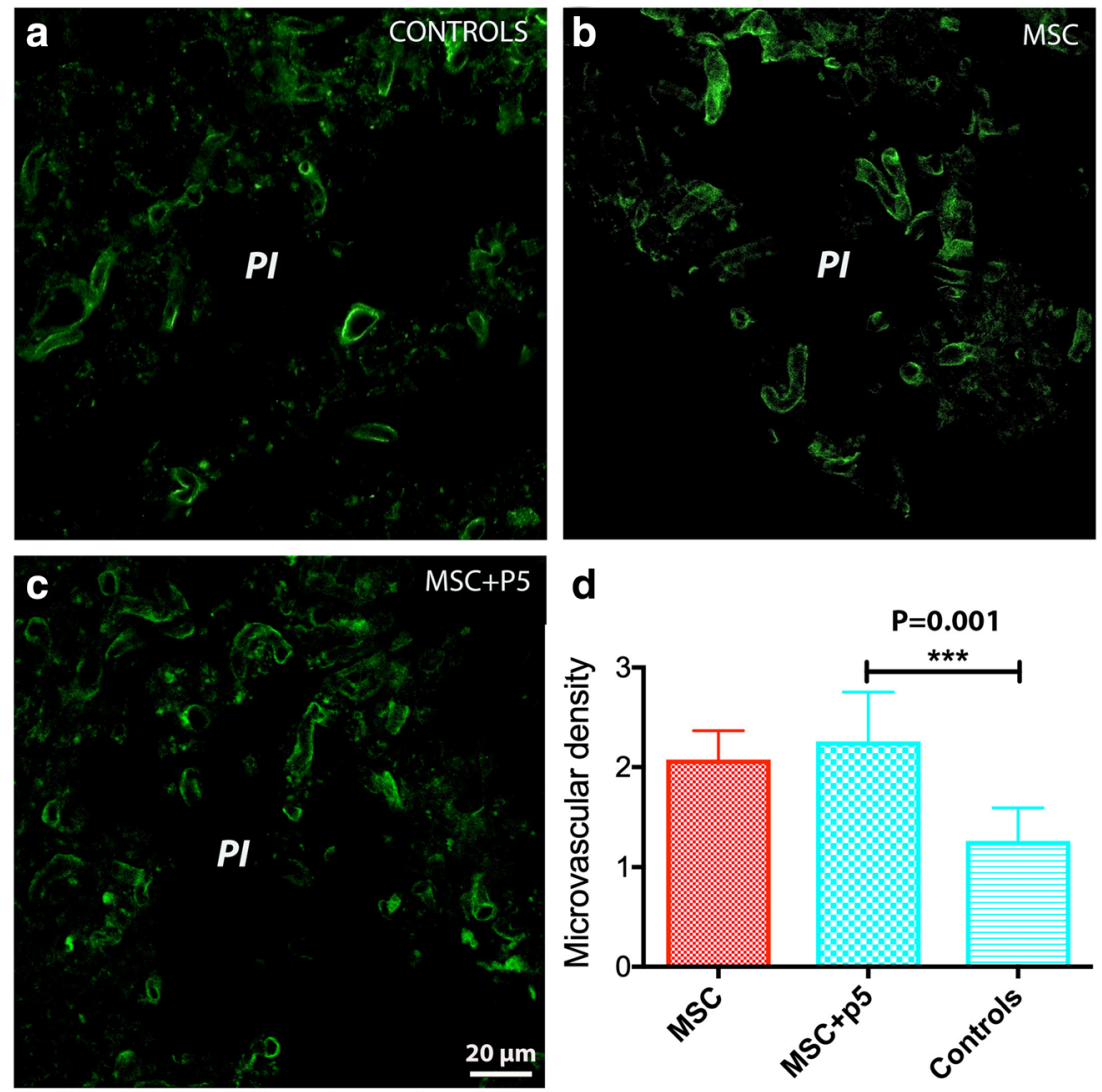

By immunostaining of the infarct area, the infarcted cortical volumes were largely similar on day 28 , suggesting that the treatment with hADMSCs + p5 was not efficacious in reducing infarct volume. However, in the peri-infarcted area of animals treated with hADMSCs $+\mathrm{p} 5$, the number of surviving transplanted cells was higher than in hADMSC-treated or control animals, suggesting primarily that the p5 peptide created conditions that supported survival of drug-loaded hADMSCs.

The underlying mechanisms could be three-fold. For one, the therapeutic peptide $\mathrm{p} 5$ could block neuronal cell apoptosis through CDK5-p35 and subsequent p53 activation [12]. In addition, the hADMSCs + p5 combination may act by reducing the number of phagocytic ANXA3/IbaI-positive cells in the peri-infarcted region. Indeed, in previous work on experimental stroke, we identified ANXA3 as a novel marker of phagocytic microglia after cerebral ischemia [24]. Therefore, we think that the therapeutic potential of p5 loading of MSCs in the clinic may be exploited in the first week post-stroke when neuroinflammation develops at its maximum and may lead to neuronal death by secondary phagocytosis.

When migrating microglia detect the "eat-me" signals, complete or partial and engulfment of these follows. This process has been coined primary phagocytosis or "phagoptosis" [25, 26]. Further, toxic neuronal insults, such as dying neurons after stroke, may cause an irreversibly exposure of the "eat-me" signal by neurons resulting in phagocytosis of dead neurons or the so-called secondary phagocytosis [27]. It is therefore a challenge to develop stroke therapies that modulate the capability of microglia to phagocytose still viable cells in the injured area after cerebral ischemia [28].

Other supportive mechanisms include stroke-stimulated increase in vascular density after stroke in rats [11]. Using an anti-collagen IV-specific marker, we found that treatment with the hADMSCs is also effective in enhancing the vascular density in the formerly infarcted area of rats during the recovery phase after stroke. However, the hADMSCs + p5 combination did not further increase the post-stroke vascular density and therefore it is unlikely that its beneficial effects on transplanted cell survival in the peri-infarcted area were due the P5 peptide.

In conclusion, administration of human adipose-derived mesenchymal stem cells (hADMSCs) loaded with the therapeutic peptide 55 to post-stroke rats led to improvement in functional recovery and created conditions that supported survival of adipose-derived, drug-loaded MSCs after cerebral ischemia, suggesting its therapeutic potential in patients with stroke and other CNS disorders [28, 29]. In addition, the double immunofluorescence with human cell-specific antibodies revealed that the number of surviving transplanted cells was higher in the peri-infarcted area of animals treated with hADMSCs + p5 than that in hADMSC-treated or control animals, concomitant with reduced number of phagocytic 
ANXA3/IbaI-positive cells in the peri-infarcted region. However, the mechanisms underlying the beneficial effects of the hADMSCs + p5 combination may not be simple and shall be investigated in a multidisciplinary study. In particular, it is not known whether hADMSCs process $\mathrm{p} 5$ into smaller neuroprotective peptides or $\mathrm{p} 5$ treatment causes hADMSCs to release neuroprotective factors.

Funding Information This study was funded by UEFISCDI, research grant PN-II-ID-PCE-2012-4-0133 to MS; project numbers PN-III-P4ID-PCE-2016-0340 to DH; and PN-III-P4-ID-PCE-2016-0215 to APW]. This work was also partially funded by the European Commission through Move-Age, an Erasmus Mundus Joint Doctorate Program (2011-0015)

\section{Compliance with Ethical Standards}

Conflict of Interest The authors declare that they have no conflict of interest.

Ethical Approval All studies with use of laboratory animals were being performed in accordance with Directive 2010/63/EU of the European Parliament and The Council of 22 September 2010 on the protection of animals used for scientific purposes with relevant Acts and Regulations. All protocols were approved by the local animal ethics committee, 8912102017. This article does not contain any studies with human participants performed by any of the authors.

Open Access This article is licensed under a Creative Commons Attribution 4.0 International License, which permits use, sharing, adaptation, distribution and reproduction in any medium or format, as long as you give appropriate credit to the original author(s) and the source, provide a link to the Creative Commons licence, and indicate if changes were made. The images or other third party material in this article are included in the article's Creative Commons licence, unless indicated otherwise in a credit line to the material. If material is not included in the article's Creative Commons licence and your intended use is not permitted by statutory regulation or exceeds the permitted use, you will need to obtain permission directly from the copyright holder. To view a copy of this licence, visit http://creativecommons.org/licenses/by/4.0/.

\section{References}

1. Griemert EV, Recarte Pelz K, Engelhard K, Schäfer MK, Thal SC. PAI-1 but not PAI-2 gene deficiency attenuates ischemic brain injury after experimental stroke. Transl Stroke Res. 2019;10(4):37280. https://doi.org/10.1007/s12975-018-0644-9.

2. Navarro-Oviedo M, Roncal C, Salicio A, Belzunce M, Rabal O, Toledo E, et al. MMP10 promotes efficient thrombolysis after ischemic stroke in mice with induced diabetes. Transl Stroke Res. 2019;10(4):389-401. https://doi.org/10.1007/s12975-018-0652-9.

3. Liu L, Yuan H, Yi Y, Koellhoffer EC, Munshi Y, Bu F, et al. Rasrelated $\mathrm{C} 3$ botulinum toxin substrate 1 promotes axonal regeneration after stroke in mice. Transl Stroke Res. 2018;9(5):506-14. https://doi.org/10.1007/s12975-018-0611-5.

4. Kim E, Yang J, Park KW, Cho S. Inhibition of VEGF signaling reduces diabetes-exacerbated brain swelling, but not infarct size, in large cerebral infarction in mice. Transl Stroke Res. 2018;9(5):540 8. https://doi.org/10.1007/s12975-017-0601-z.
5. Blecharz-Lang KG, Wagner J, Fries A, Nieminen-Kelhä M, Rösner J, Schneider UC, et al. Interleukin 6-mediated endothelial barrier disturbances can be attenuated by blockade of the IL 6 receptor expressed in brain microvascular endothelial cells. Transl Stroke Res. 2018;9(6):631-42. https://doi.org/10.1007/s12975-018-06142.

6. Chen JR, Cheng GY, Sheu CC, et al. Transplanted bone marrow stromal cells migrate, differentiate and improve motor function in rats with experimentally induced cerebral stroke. J Anat. 2008. https://doi.org/10.1111/j.1469-7580.2008.00948.x.

7. Shichinohe H, Ishihara T, Takahashi K, Tanaka Y, Miyamoto M, Yamauchi T, et al. Bone marrow stromal cells rescue ischemic brain by trophic effects and phenotypic change toward neural cells. Neurorehabil Neural Repair. 2015;29(1):80-9. https://doi.org/10. 1177/1545968314525856.

8. Deng YB, Ye WB, Hu ZZ, et al. Intravenously administered BMSCs reduce neuronal apoptosis and promote neuronal proliferation through the release of VEGF after stroke in rats. Neurol Res. 2010. https://doi.org/10.1179/174313209X414434.

9. Leu S, Lin YC, Yuen CM, et al. Adipose-derived mesenchymal stem cells markedly attenuate brain infarct size and improve neurological function in rats. J Transl Med. 2010. https://doi.org/10.1186/ 1479-5876-8-63.

10. Balseanu AT, Buga AM, Catalin B, et al. Multimodal approaches for regenerative stroke therapies: combination of granulocyte colony-stimulating factor with bone marrow mesenchymal stem cells is not superior to G-CSF alone. Front Aging Neurosci. 2014. https://doi.org/10.3389/fnagi.2014.00130.

11. Buga AM, Margaritescu C, Scholz CJ, et al. Transcriptomics of post-stroke angiogenesis in the aged brain. Front Aging Neurosci. 2014. https://doi.org/10.3389/fnagi.2014.00044.

12. Slevin M, Krupinski J. Cyclin-dependent kinase-5 targeting for ischaemic stroke. Curr Opin Pharmacol. 2009. https://doi.org/10. 1016/j.coph.2008.10.003.

13. Lee MS, Kwon YT, Li M, et al. Neurotoxicity induces cleavage of p35 to p25 by calpain. Nature. 2000. https://doi.org/10.1038/ 35012636 .

14. Zhang L, Liu W, Szumlinski KK, Lew J. p10, the N-terminal domain of $\mathrm{p} 35$, protects against $\mathrm{CDK} 5 / \mathrm{p} 25$-induced neurotoxicity. Proc Natl Acad Sci U S A. 2012. https://doi.org/10.1073/pnas. 1212914109.

15. Li Z, Fan D, Xiong D. Mesenchymal stem cells as delivery vectors for anti-tumor therapy. Stem Cell Investig. 2015;10(39782015).

16. Nava S, Sordi V, Pascucci L, et al. Long-lasting anti-inflammatory activity of human microfragmented adipose tissue. Stem Cells Int. 2019. https://doi.org/10.1155/2019/5901479.eCollection2019.

17. Lee J-H, Kim H-S, Lee S-J, Kim K-T. Stabilization and activation of p53 induced by Cdk5 contributes to neuronal cell death. J Cell Sci. 2007. https://doi.org/10.1242/jcs.03468.

18. Iyirhiaro GO, Im DS, Boonying $\mathrm{W}$, et al. Cdc25A is a critical mediator of ischemic neuronal death in vitro and in vivo. $\mathrm{J}$ Neurosci. 2017. https://doi.org/10.1523/JNEUROSCI.3017-16. 2017.

19. Pacioni S, D'Alessandris QG, Giannetti S, Morgante L, de Pascalis I, Coccè V, et al. Mesenchymal stromal cells loaded with paclitaxel induce cytotoxic damage in glioblastoma brain xenografts. Stem Cell Res Ther. 2015;6:1-11. https://doi.org/10.1186/s13287-0150185-z.

20. Fang W-H, Kumar S, McDowell G, et al. Mesenchymal stem cells loaded with $\mathrm{p} 5$, derived from CDK5 activator $\mathrm{p} 35$, inhibit calciuminduced CDK5 activation in endothelial cells. Stem Cells Int. 2016. https://doi.org/10.1155/2016/2165462.

21. Popa-Wagner A, Stöcker K, Balseanu AT, et al. Effects of granulocyte-colony stimulating factor after stroke in aged rats. Stroke. 2010. https://doi.org/10.1161/STROKEAHA.109.575621. 
22. Binukumar BK, Zheng YL, Shukla V, et al. TFP5, a peptide derived from $\mathrm{p} 35$, a Cdk5 neuronal activator, rescues cortical neurons from glucose toxicity. J Alzheimers Dis. 2014. https://doi.org/10.3233/ JAD-131784.

23. Zheng YL, Amin ND, Hu YF, et al. A 24-residue peptide (p5), derived from $\mathrm{p} 35$, the $\mathrm{Cdk} 5$ neuronal activator, specifically inhibits Cdk5-p25 hyperactivity and tau hyperphosphorylation. J Biol Chem. 2010. https://doi.org/10.1074/jbc.M110.134643.

24. Junker H, Suofu Y, Venz S, Sascau M, Herndon JG, Kessler C, et al. Proteomic identification of an upregulated isoform of annexin A3 in the rat brain following reversible cerebral ischemia. Glia. 2007;55: 1630-7.

25. Fuhrmann M, Bittner T, Jung CKE, et al. Microglial Cx3cr1 knockout prevents neuron loss in a mouse model of Alzheimer's disease. Nat Neurosci. 2010. https://doi.org/10.1002/glia.20581.
26. Neher JJ, Neniskyte U, Zhao J-W, et al. Inhibition of microglial phagocytosis is sufficient to prevent inflammatory neuronal death. J Immunol. 2011. https://doi.org/10.4049/jimmunol.1003600.

27. Brown GC, Neher JJ. Microglial phagocytosis of live neurons. Nat Rev Neurosci. 2014. https://doi.org/10.1038/nrn3710.

28. Stonesifer C, Corey S, Ghanekar S, et al. Stem cell therapy for abrogating stroke-induced neuroinflammation and relevant secondary cell death mechanisms. Prog Neurobiol. 2017. https://doi.org/ 10.1016/j.pneurobio.2017.07.004.

29. Lee J-Y, Lin R, Nguyen H, Grant Liska M, Lippert T, Kaneko Y, et al. Histopathological and behavioral assessments of aging effects on stem cell transplants in an experimental traumatic brain injury. Methods Mol Biol. 2019. https://doi.org/10.1007/7651_2018_121.

Publisher's Note Springer Nature remains neutral with regard to jurisdictional claims in published maps and institutional affiliations. 